\title{
A novel adriamycin analogue derived from marine microbes induces apoptosis by blocking Akt activation in human breast cancer cells
}

\author{
JIE YUAN ${ }^{1,2}$, ZHENJIAN HE $^{1}$, JUEHENG WU $^{2}$, YONGCHENG LIN ${ }^{2,3}$ and XUN ZHU ${ }^{1,2}$ \\ ${ }^{1}$ Key Laboratory of Tropical Disease Control (Sun Yat-Sen University), Ministry of Education; ${ }^{2}$ Guangdong Province Key \\ Laboratory of Functional Molecules in Oceanic Microorganism (Sun Yat-Sen University), Bureau of Education; \\ ${ }^{3}$ School of Chemistry and Chemical Engineering, Sun Yat-Sen University, Guangzhou 510080, P.R. China
}

Received October 12, 2010; Accepted January 20, 2011

DOI: $10.3892 / \mathrm{mmr} .2011 .434$

\begin{abstract}
P-3 is a novel anthracenedione derivative isolated from the secondary metabolites of endophytic fungus from the South China Sea. In previous studies, 1403P-3 was found to exhibit potent cytotoxicity against human cancer cells, but its molecular target and the mechanisms mediating its cytotoxicity remain unknown. In this study, we showed that 1403P-3 markedly inhibited the survival of the human breast cancer cells MCF-7 and MDA-MB-435 in a dose-dependent manner, with an $\mathrm{IC}_{50}$ of 9.5 and $7.6 \mu \mathrm{M}$, respectively. Apoptosis induced by $1403 \mathrm{P}-3$ was detected, as indicated by Annexin V-FITC/PI staining, elevated activated caspase-8 and -9 , and cleavaged PARP determined by Western blot analysis. It is of note that the phosphorylation level of Akt was significantly reduced in 1403P-3-treated cells in a dose- and time-dependent manner. Taken together, our data demonstrated that 1403P-3 induced breast cancer cell apoptosis by blocking Akt activation, suggesting that 1403P-3 may be a promising candidate compound for anti-tumor drug development.
\end{abstract}

Correspondence to: Dr Xun Zhu, Zhongshan School of Medicine, Sun Yat-Sen University, 74 Zhongshan Road II, Guangzhou, Guangdong 510080, P.R. China

E-mail: zhuxun333@gmail.com

Abbreviations: DMEM, Dulbecco's modified Eagle's medium; DMSO, dimethylsulphoxide; FITC, fluorescein isothiocyanate; PI, propidium iodide; PARP, poly (ADP-ribose) polymerase; MTT, 3-(4,5-dimethylthiazol-2-yl)-2,5-diphenyl tetrazolium bromide; PBS, phosphate-buffered saline; IETD-pNA, L-isoleucyl-L-glutamylL-Threonyl-L-aspartic-p-nitroanilide acid amide; LEHD-pNA, L-leucine-Lglutamyl-L-histidyl-L-aspartic-p-nitroaniline acid amide; TUNEL, terminal deoxynucleotidyl transferase-mediated dUTP nick-end labelling

Key words: marine, adriamycin analogue, breast cancer, apoptosis, Akt

\section{Introduction}

Breast cancer is the most common malignant disease in women and the second most frequent cancer in the world, with a rising incidence. On average, the number of diagnosed breast cancer patients increases by 1.05 million annually, accounting for $22 \%$ of all new cancer cases worldwide, and more than 410,000 women die from the disease each year $(1,2)$.

It is widely acknowledged that disordered regulation of apoptosis is a major mechanism of tumorigenesis and drug resistance $(3,4)$. During the apoptotic process, pro-apoptotic inducers trigger a cascade of reactions that eventually lead to the cleavage of poly (ADP-ribose) polymerase (PARP) into 89and $24-\mathrm{kDa}$ fragments, which respectively contain the active site and the DNA-binding domain of the enzyme, leading to the apoptotic death of a variety of cell types $(5,6)$.

The phosphorylation of Akt modulates multiple molecular targets involved in the inhibition of apoptosis, cell growth and metabolism. In general, the decrease of Akt phosphorylation causes cells to undergo apoptosis, cell cycle arrest or homeostasis under low nutrient conditions. Moreover, the activation of the PI3K/Akt signaling pathway confers resistance to many types of cancer therapy and is a poor prognostic factor for numerous neoplastic lesions, making Akt a promising target for innovative cancer treatment (7).

Anthracyclines represent an important class of anti-cancer chemotherapeutic drugs, and have shown a wide spectrum of anti-cancer activities $(8,9)$. As a novel adriamycin analogue derived from marine microbes, 1403P-3 has been shown to cause the apoptosis of KB cells and MDR KBv200 oral carcinoma cells (10). The mechanism underlying such anti-cancer effects of 1403P-3, as well as its effects on other cancer types, are not clear. The present study was performed to investigate the potential role of $1403 \mathrm{P}-3$ in the growth inhibition of breast cancer cells, and to characterize the signaling pathway affected by 1403P-3 using a breast cancer model.

\section{Materials and methods}

Chemicals and reagents. Pure 1403P-3 compound was prepared and purified as described previously (11). Before use, 
the compound was initially dissolved in $0.5 \%(\mathrm{v} / \mathrm{v})$ dimethylsulphoxide (DMSO) at $1 \mathrm{mM}$, and was then diluted in cell culture medium according to experimental requirements.

Cell lines and cell culture. The human breast cancer cell line MDA-MB-435 was cultured in Dulbecco's modified Eagle's medium (DMEM; Invitrogen, Carlsbad, CA, USA) supplemented with 5\% fetal bovine serum (Hyclone, Logan, UT, USA), $2 \mathrm{mM}$ L-glutamine, $100 \mathrm{mg} / \mathrm{ml}$ streptomycin and $100 \mathrm{U} / \mathrm{ml}$ penicillin (Invitrogen). Cells were grown at $37^{\circ} \mathrm{C}$ in a humidified $5 \% \mathrm{CO}_{2}$ atmosphere.

MTT assay for cell viability assessment. The dose-dependent effect of 1403P-3 on the viability of the human breast cancer cell line MDA-MB-435 was evaluated with varying concentrations $(0,0.1,1,10$ and $100 \mu \mathrm{M})$ of $1403 \mathrm{P}-3$ treatment using the MTT assay. The MTT assay is based on the conversion of a yellow substrate to dark blue formazan by mitochondrial dehydrogenases in living cells. In this assay, cells were incubated with fresh medium containing MTT (final concentration $1 \mathrm{mg}$ / $\mathrm{ml}$ ) for $4 \mathrm{~h}$ at $37^{\circ} \mathrm{C}$. The dark blue formazan crystals formed in intact cells were solubilized with DMSO (Sangon Biotech, Shanghai, China) and the absorbance was measured at $570 \mathrm{~nm}$ with a reference wavelength of $630 \mathrm{~nm}$ using a microplate reader (BioTek Synergy2; BioTek, Winooski, VT, USA). The percentage cell growth inhibition rate was calculated using the formula: Inhibition rate $(\%)=[(1-\mathrm{OD}$ of treated cells $) / \mathrm{OD}$ of control cells] x $100 \%$, where OD stands for the optical density (12). The half maximal inhibitory concentration $\left(\mathrm{IC}_{50}\right)$ was calculated using Bliss's software (13).

Annexin V-FITC/PI dual staining. Cells were grown for 2-3 days prior to drug treatment [final DMSO concentration, $0.5 \%(\mathrm{v} / \mathrm{v})]$, then maintained at $37^{\circ} \mathrm{C}$ for the indicated additional time periods. According to the manufacturer's protocol (Keygen Biotech, Nanjng, China), the cells were washed twice with PBS and incubated in $500 \mathrm{ml}$ binding buffer containing Annexin V-FITC and PI. Then, the cells were left standing for $10 \mathrm{~min}$ at $22^{\circ} \mathrm{C}$ in the dark. The chamber slide was washed twice with PBS, then the specimen was air-dried and a coverslip was applied with antifade mounting solution. The cells were examined with an inverted microscope (Zeiss Axiovert 100M; Carl Zeiss, Germany) with epifluorescence and appropriate filters. Ten randomly selected microscopic fields were captured. Experiments were performed in triplicate.

Caspase activity assay. The activity of caspase- 8 and -9 was measured using a caspase colorimetric assay (Keygen Biotech). After treatment with 1403P-3 at different concentrations for $48 \mathrm{~h}$, the cells were harvested, washed with PBS and then resuspended in chilled lysis buffer. Following incubation on ice for $40 \mathrm{~min}$, the cells were centrifuged to collect the supernatant and protein concentrations were determined using the Bradford protein assay (Keygen Biotech). Subsequently, $150 \mathrm{mg}$ of each protein sample was diluted with $50 \mathrm{ml}$ lysis buffer and added to $50 \mathrm{ml}$ of $2 \mathrm{X}$ reaction buffer containing $10 \mathrm{mM}$ dithiothreitol in a 96-well plate. A colorigenic substrate $(5 \mu \mathrm{l})$, IETD-pNA (L-isoleucyl-L-glutamyl-LThreonyl-L-aspartic-p-nitroanilide acid amide) or LEHD-pNA (L-leucine-L-glutamyl-L-histidylL-aspartic-p-nitroaniline acid amide), was added to each well,

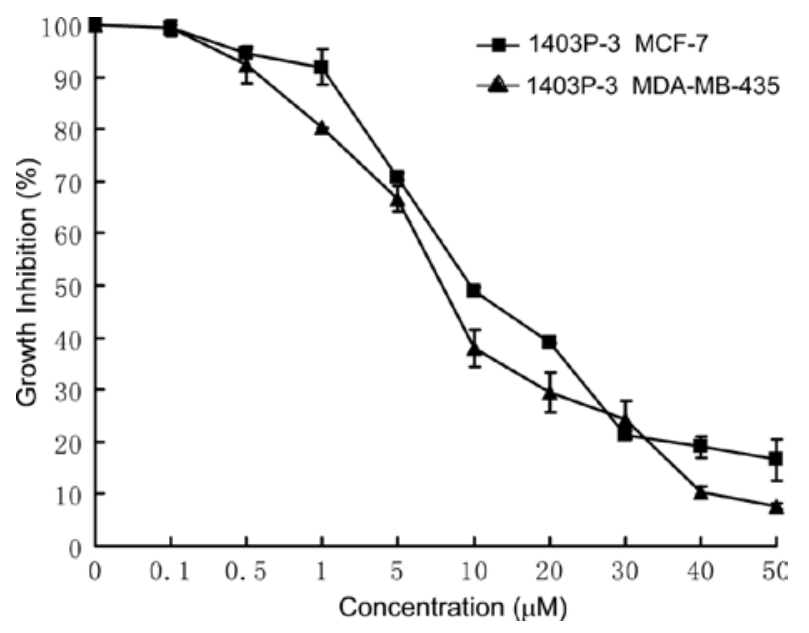

Figure 1. Growth inhibitory effect of 1403P-3 treatment at the indicated concentrations for $24 \mathrm{~h}$ on the human breast cancer cell line MDA-MB-435. Cell viability was measured by the MTT assay. Data were obtained from three separate experiments conducted with triplicate samples. Statistical analysis used the Student's t-test.

and the plate was incubated at $37^{\circ} \mathrm{C}$ in the dark for $4 \mathrm{~h}$. ODs were determined at $405 \mathrm{~nm}$ using a microplate reader (BioTek Synergy2; BioTek).

Western blot analysis. Cells were harvested in sampling buffer [62.5 mmol/1 Tris- $\mathrm{HCl}$ (pH 6.8), 10\% glycerol and 2\% SDS] and heated for $5 \mathrm{~min}$ at $100^{\circ} \mathrm{C}$. The protein concentration was determined by the Bradford assay using a commercial kit purchased from Bio-Rad Laboratories (Hercules, CA, USA). Equal quantities of protein were separated electrophoretically on $10 \%$ SDS/polyacrylamide gels and transferred onto polyvinylidene difluoride membranes (Roche, Mannheim, Germany). The membrane was probed with rabbit anti-human PARP, rabbit anti-human phospho-Akt (ser473) or rabbit antihuman Akt antibodies (Cell Signaling Technology, Beverly, MA, USA). The expression of phospho-Akt was determined with horseradish peroxidase-conjugated anti-rabbit immunoglobulin G $(1: 3,000)$ and enhanced chemiluminescence (Pierce, Rockford, IL, USA) according to the manufacturer's suggested protocols. The membranes were stripped and re-probed with an anti-Actin mouse monoclonal antibody (Sigma, St. Louis, MO, USA) as a loading control.

Statistical analysis. Statistical analyses were carried out using the SPSS 10.0 statistical software package. The data are expressed as the means \pm SD. For the MTT assay, statistical analyses were performed using Student's t-test. The statistical significance of the differences between groups was determined by one-way analysis of variance (ANOVA) followed by a Newman-Keuls post hoc test. $\mathrm{P} \leq 0.05$ was considered statistically significant.

\section{Results}

Inhibitory effect of 1403P-3 on human breast cancer $M D A-M B-435$ cell growth in vitro. It has been reported that 1403P-3 exert potent cytotoxicity against oral squamous carcinoma KB and KBv200 cells (10). To further investigate its 
A

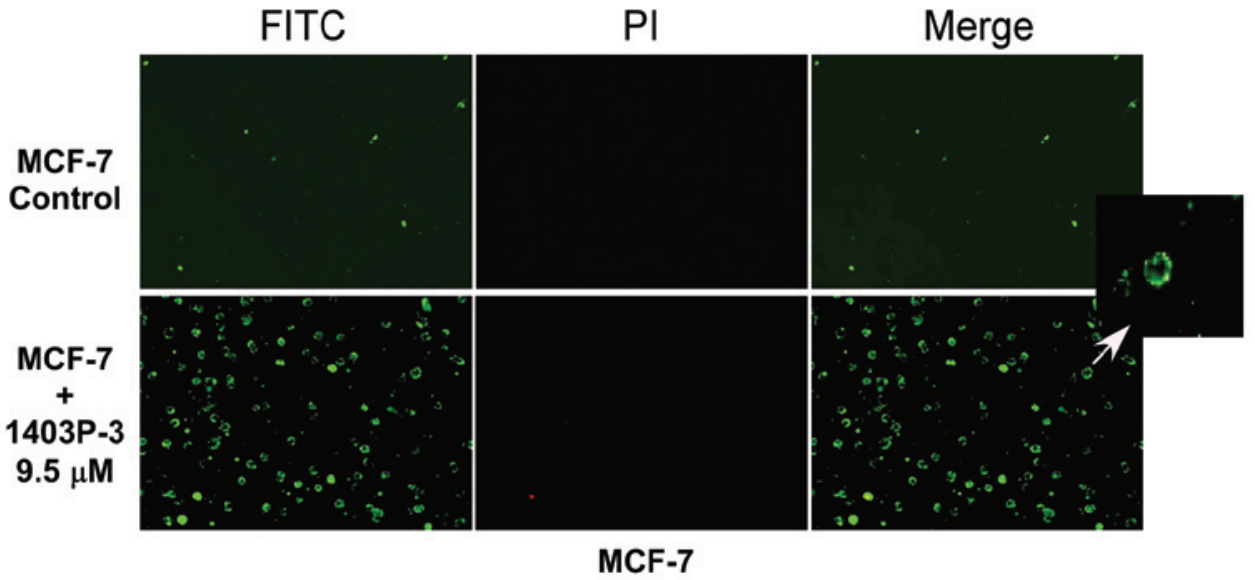

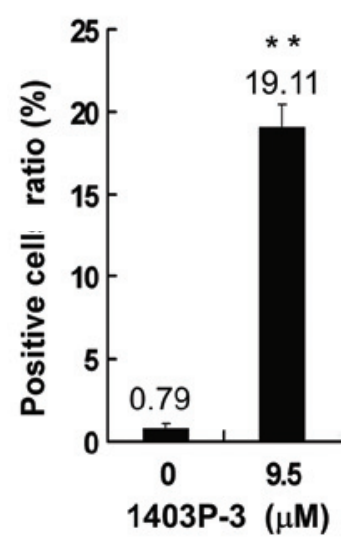

Figure 2. Induction of apoptosis of MCF-7 breast cancer cells treated with 1403P-3, detected by Annexin V-FITC/PI staining. (A) Cells were exposed to $9.5 \mu \mathrm{M}$ 1403P-3 for $8 \mathrm{~h}$. The apoptotic cells were Annexin V-FITC + PI- (green) and the dead cells were labeled with PI (red). Original magnification, $\mathrm{x} 200$. Ten representative stained fields for each section were analyzed to determine the apoptotic index. (B) Apoptotic index as determined by counting and calculating the percentage of FITC-positive cells in ten fields. Data for the quantitative assessment of apoptosis are expressed as the means \pm SD. ${ }^{* *} \mathrm{P}<0.01$ compared to the control. FITC, fluorescein isothiocyanate; PI, propidium iodide.

A

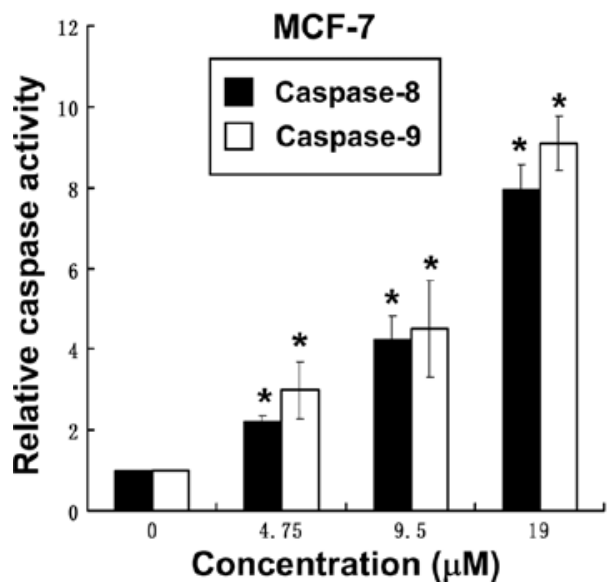

B

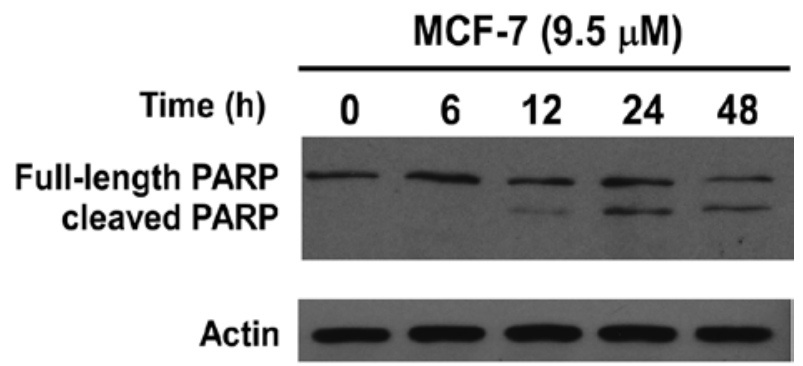

Figure 3. Activation of caspase-8 and -9 and poly (ADP-ribose) polymerase (PARP) in MCF-7 cells treated with 1403P-3. (A) Enzymatic activity of caspase-8 and -9 in MCF-7 cells treated for $24 \mathrm{~h}$ with 1403P-3 at the indicated concentrations, as assessed by colorimetric assay. Fold increases in the activity of caspase- 8 and -9 were determined by comparison to those of the vehicle-treated control cells. Results are presented as the means \pm SD. ${ }^{*}<0.05$ significant differences compared to the control. (B) Effect of 1403P-3 on the cleavage of PARP in MCF-7 cells, as analyzed by Western blotting. Cells were exposed to $9.5 \mu \mathrm{M}$ of $1403 \mathrm{P}-3$ for the indicated times and the total cell lysates were analyzed for proteolytic cleavage of PARP. Actin was used as a loading control.

anti-tumor effects in human breast cancer, the cytotoxicity of 1403P-3 was measured by the MTT assay. As shown in Fig. 1, 1403P-3 exhibited a marked inhibition of the survival of MCF-7 and MDA-MB-435 cells in a dose-dependent manner. The $\mathrm{IC}_{50}$ value was 9.7 $\mu \mathrm{M}$ for MCF-7 and 7.6 $\mu \mathrm{M}$ for MDA-MB-435.

Induction of an irreversible apoptotic process by $1403 P-3$ in human breast cancer cells. To determine whether the observed decrease in the survival of breast cancer cells was due to the induction of apoptosis, MCF-7 breast cancer cells were treated with $1403 \mathrm{P}-3$ at a concentration of $\mathrm{IC}_{50} \sim 9.5 \mu \mathrm{M}$ for $8 \mathrm{~h}$. The apoptosis of the cells was detected by Annexin V-FITC/PI dual staining. The results showed that the treatment with $1403 \mathrm{P}-3$ induced apoptosis, exhibiting a ratio of Annexin V-FITC + PI cells of $19.11 \%$, which was significantly higher than that of the control cells $(\mathrm{P}<0.01)$ (Fig. 2).

To further confirm the above-mentioned observation, we characterized the effect of 1403P-3 on apoptosis protec- tion by assessing its impact on the enzymatic activation of caspase- 8 and -9 and the cleavage of PARP proteins, which are critical for cell apoptosis. The activity of caspase- 8 and -9 was measured by a caspase colorimetric assay. The enzymatic activity of the caspases showed a dose-dependent increase in conjuction with 1403P-3 treatment (Fig. 3A). Concurrently, the cleavage of PARP in the 1403P-3-treated MCF-7 cells was detectable by Western blotting. Cleavage of PARP from 116 to $85 \mathrm{kDa}$ was clearly demonstrated in the MCF-7 cells exposed to 1403P-3 (Fig. 3B). Collectively, these data suggest that $1403 \mathrm{P}-3$ induces apoptosis in human breast cancer cells.

1403P-3 reduced Akt phosphorylation levels in human breast cancer cells. To investigate the signaling cascade leading to apoptosis induced by 1403P-3 in breast cancer cells, changes in the the phosphorylation levels of Akt in cells treated with 1403P-3 were examined. As shown in Fig. 4, 1403P-3 treatment resulted in a dose- and time-dependent decrease in 


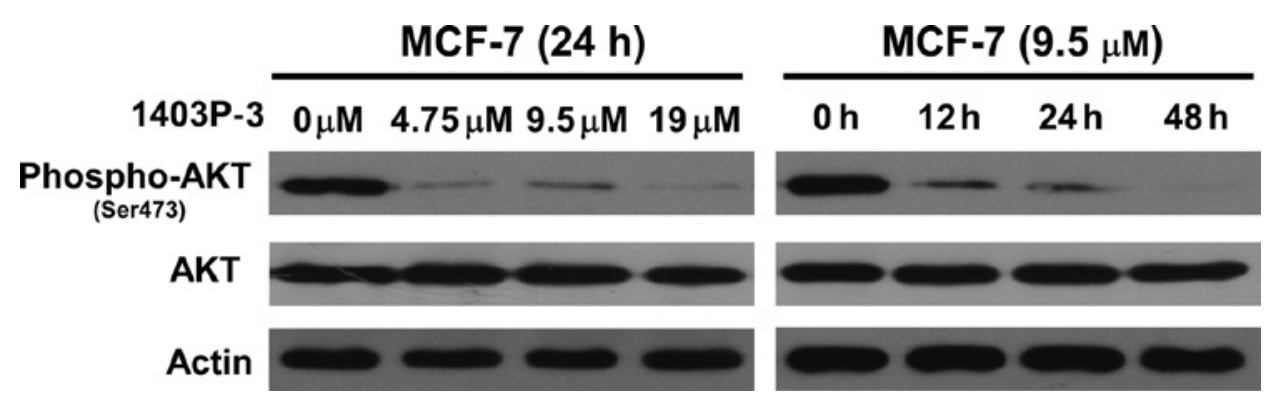

Figure 4. 1403P-3-induced apoptosis was accompanied by decreased phosphorylation of Akt proteins in a dose- and time-dependent manner in breast cancer cells. After cells were treated with indicated concentrations of 1403P-3 for $48 \mathrm{~h}$, the whole-cell lysates were assayed by Western blotting and corresponding antibodies. The significant reduction of the phosphorylation level of Akt at Ser473 site was observed in MCF-7 cells with the total Akt unchanged.

phospho-Ser473-Akt in the MCF-7 cells, while the expression of total Akt remained unchanged. Together, these data indicate that 1403P-3 inactivated Akt, which in turn may modulate the downstream pathways and target genes, finally triggering apoptosis.

\section{Discussion}

Although a thorough understanding of apoptotic defects and clinical multidrug resistance remains to be developed (14), the hypothesis for the causal relationship between apoptosis and drug-induced cytotoxicity has been widely accepted (3). Developing an apoptosis-inducing new drug represents a promising direction in anti-cancer therapy. Unfortunately, many tumor types evade drug-induced death signals (15). Among the several apoptosis-related signaling molecules, Akt has been suggested to be an important modulator in sustaining survival against the apoptosis of cancer cells, and its function is frequently found altered in a variety of human cancers $(16,17)$. It has been shown that the up-regulation of Akt is a common event in many human cancer types, and that the activation of the PI3K/Akt pathway contributes to chemotherapeutic resistance in human cancers (18-20). Therefore, studying the mechanism of the blockage of Akt activation may reveal promising targets for anti-cancer therapy.

To date, chemotherapy has played an important role in breast cancer treatment. Adriamycin and other anthracyclines are currently prefered among treatments for breast cancer. However, multidrug resistance and severely adverse side effects (e.g., cardiotoxicity and myelosuppression) limit the use of anthracycline $(21,22)$. The development of new chemotherapeutic agents with improved pharmacological properties is essential (23).

1403P-3 is a novel compound derived from the secondary metabolites of the mangrove endophytic fungus no. 1403 from the South China Sea, and is an anthracenedione derivative (11). As the present data show, 1403P-3 possesses a significant inhibitory effect on the survival of human breast cancer cells. Notably, the phosphorylation level of Akt was significantly reduced in 1403P-3-treated cells, and apoptotic morphological changes occurred during the exposure of breast cells to 1403P-3. Although the exact target triggering the apoptosis of breast cancer cells requires further study, our data suggest that 1403P-3 is a candidate compound that merits a thorough preclinical evaluation and mechanistic studies in the laboratory.

\section{Acknowledgements}

This study was supported by the 863 Project (2007AA09Z448, 2007AA09Z431), the National Natural Science Foundation of China (40806059), the Natural Science Foundation of Guangdong Province, China (8151008901000014), the Key Project of Science \& Technology Planning of Guangdong Province (2007A03260001), China's Marine Commonwealth Research Project (201005022-2) and the Basic Research Project of the Ministry of Science and Technology, China (2007FY210600).

\section{References}

1. Parkin DM: Global cancer statistics in the year 2000. Lancet Oncol 2: 532-542, 2001

2. Coughlin SS and Ekwueme DU: Breast cancer as a global health concern. Cancer Epidemiol 33: 315-318, 2009.

3. Johnstone RW, Ruefli AA and Lowe SW: Apoptosis: a link between cancer genetics and chemotherapy. Cell 108: 153-164, 2002.

4. Houghton JA: Apoptosis and drug response. Curr Opin Oncol 11: 475-481, 2002.

5. Tewari M, Quan LT, O'Rourke K, Desnoyers S, Zeng Z, Beidler DR, Poirier GG, Salvesen GS and Dixit VM: Yama/ CPP32 beta, a mammalian homolog of CED-3, is a CrmAinhibitable protease that cleaves the death substrate poly (ADP-ribose) polymerase. Cell 81: 801-809, 1995.

6. Kaufmann SH, Desnoyers S, Ottaviano Y, Davidson NE and Poirier GG: Specific proteolytic cleavage of poly (ADP-ribose) polymerase: an early marker of chemotherapy-induced apoptosis. Cancer Res 53: 3976-3985, 1993.

7. Lindsley CW, Barnett SF, Layton ME and Bilodeau MT: The PI3K/Akt pathway: recent progress in the development of ATP-competitive and allosteric Akt kinase inhibitors. Curr Cancer Drug Targets 8: 7-18, 2008.

8. Hortobágyi GN: Anthracyclines in the treatment of cancer. An overview. Drugs 54 (Suppl 4): 1-7, 1997.

9. Cortés-Funes $\mathrm{H}$ and Coronado C: Role of anthracyclines in the era of targeted therapy. Cardiovasc Toxicol 7: 56-60, 2007.

10. Zhang JY, Wu HY, Xia XK, Liang YJ, Yan YY, She ZG, Lin YC and Fu LW: Anthracenedione derivative 1403P-3 induces apoptosis in KB and KBv200 cells via reactive oxygen speciesindependent mitochondrial pathway and death receptor pathway. Cancer Biol Ther 6: 1413-1421, 2007.

11. Jiang GC, Lin YC, Zhou SN, Vrumoed LLP and Jones EBG: Studies on the secondary metabolites of mangrove fungus no. 1403 from the South China Sea. Acta Scientiarum Naturalium Universitatis Sun Yat-sen 39: 68-72, 2000.

12. Moon IJ, Choi K, Choi YK, Kim JE, Lee Y, Schreiber AD and Park JG: Potent growth inhibition of leukemic cells by novel ribbon-type antisense oligonucleotides to c-myb1. J Biol Chem 275: 4647-4653, 2000.

13. Bliss C: The calculation of the dose-mortality curve. Ann Appl Biol 22: 134-167, 1935. 
14. Brown JM and Wouters BG: Apoptosis, p53, and tumor cell sensitivity to anticancer agents. Cancer Res 59: 1391-1399, 1999.

15. Igney FH and Krammer PH: Death and anti-death: tumour resistance to apoptosis. Nat Rev Cancer 2: 277-288, 2002.

16. Plas DR and Thompson CB: Akt-dependent transformation: there is more to growth than just surviving. Oncogene 24: 7435-7442, 2005.

17. Yoeli-Lerner $\mathrm{M}$ and Toker A: Akt/PKB signaling in cancer: function in cell motility and invasion. Cell Cycle 5: 603-605, 2006.

18. Lee S, Choi EJ, Jin C and Kim DH: Activation of PI3K/Akt pathway by PTEN reduction and PIK3CA mRNA amplification contributes to cisplatin resistance in an ovarian cancer cell line. Gynecol Oncol 97: 26-34, 2005

19. LoPiccolo J, Granville CA, Gills JJ and Dennis PA: Targeting Akt in cancer therapy. Anticancer Drugs 8: 861-874, 2007.
20. Lu S, Ren C, Liu Y and Epner DE: PI3K-Akt signaling is involved in the regulation of p21 (WAF/CIP) expression and androgenindependent growth in prostate cancer cells. Int J Oncol 28: 245-251, 2006

21. Schimmel KJ, Richel DJ, van den Brink RB and Guchelaar HJ: Cardiotoxicity of cytotoxic drugs. Cancer Treat Rev 30: 181-191, 2004.

22. Zucchi R and Danesi R: Cardiac toxicity of antineoplastic anthracyclines. Curr Med Chem Anticancer Agents 3: 151-171, 2003.

23. Binaschi M, Bigioni M, Cipollone A, Rossi C, Goso C, Maggi CA, Capranico G and Animati F: Anthracyclines: selected new developments. Curr Med Chem Anticancer Agents 1: 113-130, 2001 